\title{
RELIABILITY AND SENSITIVITY ANALYSIS OF A CONDITION MONITORING TECHNIQUE
}

\author{
Teresia RAMDÉN, Petter KRUS and Jan-Ove PALMBERG \\ Division of Fluid Power Technology \\ Department of Mechanical Engineering \\ Linköping University, S-581 83 Linköping, Sweden \\ terra@ikp.liu.se \\ http://hydra.ikp.liu.se
}

\begin{abstract}
A condition monitoring technique based on vibration measurements and evaluation by means of neural networks has previously been investigated. Preliminary results were promising. In this work, the reliability and the sensitivity of the method are discussed. This study has used a simulation approach to study the sensitivity. An experimentally verified simulation model which yields the internal forces in the pump has been used in combination with experimentally found transfer functions to find the vibration on the surface of the pump housing. Different designs of the valve plate have been studied to simulate an increased internal leakage in the pump. For the evaluation of simulation or measurement results, neural networks are used.
\end{abstract}

\section{KEYWORDS}

Condition monitoring, Neural networks, Pumps, Vibrations, Simulation

\section{NOMENCLATURE}

$a_{i} \quad$ activation for neuron $i$

$f_{i} \quad$ nonlinear sigmoidal transfer function for neuron $i$

$i_{i} \quad$ input of neuron $i$ (in input layer)

$o_{i} \quad$ output of neuron $i$

$w_{i j} \quad$ variable weight from the $i$ th input to the $j$ th neuron

\section{INTRODUCTION}

In condition monitoring it is always difficult to decide which parameters to measure and how to evaluate the signals. The more parameters that are measured, the more accurately can we determine the condition of a system or a component. However, measuring more parameters using more sensors, leads to increasing requirements on the evaluation system. Thus, there is, for simplicity and economical reasons, a desire to find the key parameters which give the relevant information. It is reasonable to assume that the monitoring of only one component could be carried out by using fewer parameters than what would be required when investigating a complete system. The assumption made in this paper, is that it should even be possible to use only one parameter, e $g$ the vibration signal, to monitor a fluid power pump. The vibration signal has turned out to be a relevant signal to use for fault detection. In this capacity it surpasses traditional indicators such as pressure, flow and temperature [1]. Other reasons for choosing vibration measurements are that it is possible to do them on-line, that they do not require any changes to the system, and, nowadays, the availability of cheap and sufficiently accurate sensors.

An approach for a condition monitoring technique based on vibration measurements was discussed in earlier works by the authors, e $g[2,3]$. However, in these articles, the emphasis has been on developing 


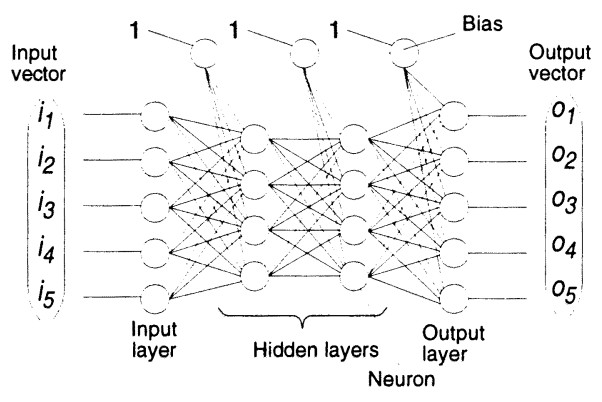

Figure 1. A three-layer neural network.

the technique, not to discuss or investigate its reliability and sensitivity to changes in operating condition etc. It is, though, extremely important to know how valid these results are when it comes to a real situation.

\section{CONDITION MONITORING TECHNIQUE}

This condition monitoring technique is based on knowledge of the behaviour of the pump (or other system) to be monitored in both original, faultless condition and in the most common faulty conditions. In the case of a pump, measurements are made on several pumps of the same type for these conditions beforehand. The reason for choosing to measure on more than one pump is that it is necessary to ignore differences between individual pumps. Results from these measurements are used to train a neural network (see section Neural networks below). When running the pump, new measurements are made during equal conditions, and the trained neural network is used to evaluate the results.

The measurements mentioned in the last paragraph, may be measurements of different parameters as stated in the introduction, but in the case described here it has always been vibration measurements on the surface of the pump. The measurements are made in the time domain with a PC equipped with an analogue to digital converter board. The frequency spectrum of the vibration is calculated by means of the FFT algorithm. Since all the measurements are made at the same rotational speed, it is possible to find harmonics in the spectrum. These harmonics are the properties that are used for the condition monitoring.

\section{Neural networks}

The condition monitoring approach used in this paper, uses neural networks for the measurement evaluation, see e $\mathrm{g}[4,5]$. A neural network is a net consisting of neurons, connected to each other in layers, as shown in fig. 1. A net consists of one input layer, one

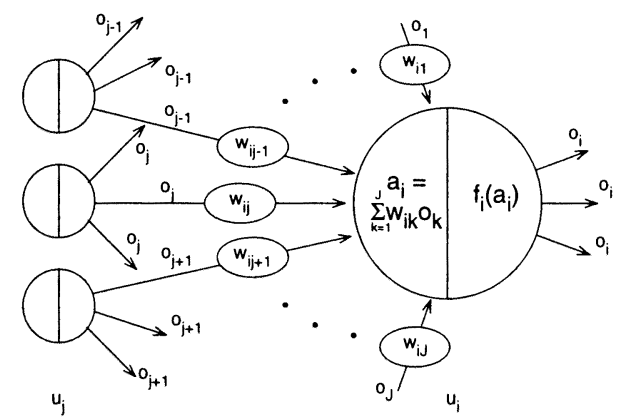

Figure 2. A neuron with inputs from the previous layer.

or more hidden layers and one output layer, and it is said to be a two-layer network if it has one hidden and one output layer. The outputs from the neurons in one layer are inputs to all the neurons in the next layer, fig. 1. Often, a layer can have a bias as well, as indicated in the figure. One neuron can be described as in fig. 2. The neuron receives inputs from neurons in the previous layer. The inputs are multiplied by separate weights and then summed to become the input of the transfer function of this neuron. This function may be e $\mathrm{g}$ the sigmoid function from -1 to 1 , the sigmoid function from 0 to 1 or just a linear function as in eq. 1, 2 and 3, respectively.

$$
\begin{gathered}
f_{i}\left(a_{i}\right)=\frac{1-e^{-2 a_{i}}}{1+e^{-2 a_{i}}} \\
f_{i}\left(a_{i}\right)=\frac{1}{1+e^{-2 a_{i}}} \\
f_{i}\left(a_{i}\right)=a_{i}
\end{gathered}
$$

The result from this transfer function is the output of the neuron, and it is passed on to the neurons in the next layer of the net.

The objective is to adjust the weights to minimise the output error. The error is the difference between the actual output for one specific input vector and the desired output for this input. The error to be minimised is most often the sum of the squared errors. This minimisation is done by any suitable training technique, very often the back-propagation method (which is the one used here), but any optimisation algorithm may actually be used.

\section{Neural networks in this application}

In this particular case, the input vector will consist of chosen values from the vibration time signal and from the amplitude and phase of the frequency spectrum. It is possible to look at the amplitude and phase because all the simulations (or measurements) are made at the same rotational speed. The choice of values should preferably be performed by an automatic preprocessor, as in [1]. It has not been so in this case 


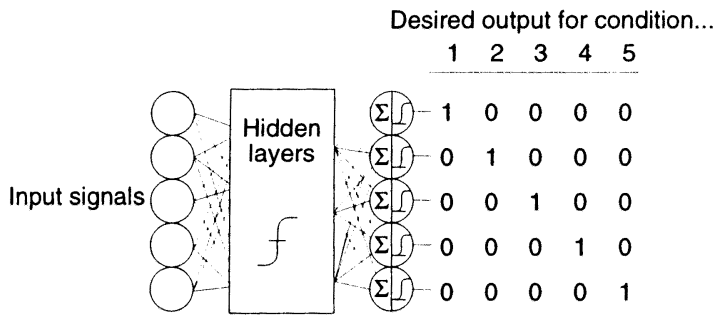

Figure 3. Desired output vectors for input vectors from five different conditions.

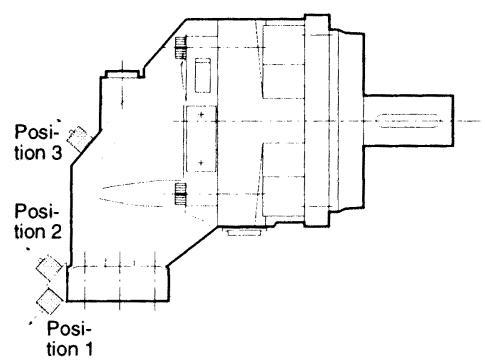

Figure 4. VOAC F12 pump and the accelerometers.

which is described later on in section Simulations and evaluation.

The output vector will, ideally, consist of the value " 1 " for one of the output neurons and the value " 0 " for all the others. There will be as many neurons in the output layer as there are different conditions to be distinguished. In fig. 3 , the desired output vectors from a net that is to identify five conditions are shown.

Here, we have used the Matlab Neural Network Toolbox [5]. The training has been done with the back-propagation algorithm, with momentum and adaptive learning rate to increase the performance of the method. The momentum method will reduce the risk of getting stuck in shallow minima, and the adaptive learning rate attempts to keep the learning step size as large as possible, while still keeping the learning stable. See the Neural Network Toolbox Manual [5] for a further description of these methods.

\section{SYSTEM TO BE MONITORED}

The pump that has been used in this study is a fixeddisplacement bent-axis piston pump of type VOAC Hydraulic F12-40, see fig. 4. It has seven cylinders and a displacement of $40 \mathrm{~cm}^{3} / \mathrm{rev}$ and a power range of up to approximately $85 \mathrm{~kW}$ (continuously). In the previous works, measurements have been done with two different accelerometers: one simple, robust sensor, especially developed for the automobile industry and thus quite cheap, and one more conven- tional. Both sensors are piezoelectric vibration sensors, and the first one has fairly non-linear characteristics, whereas the second has rather linear. The main aim is to be able to use the cheaper one and the other one is used for evaluation of the first. Now, three sensors of the conventional type have been used, at three different positions of the pump as indicated in fig. 4 .

\section{Simulation model}

In a parallel project [6], an accurate simulation model of the VOAC F12 pump has been developed. The model is made in the HOPSAN simulation package [7] developed at Linköping University. The pump model can calculate the internal forces in the pump, together with pump pulsations. By means of experimentally found transfer functions, it is possible to find the acceleration of the pump housing from the internal forces. The simulation model has been extensively verified experimentally in [6].

The pump simulation model has made it possible to do this sensitivity study by means of simulation instead of experimental work. Of course, these results should later on be verified experimentally.

\section{SIMULATIONS AND EVALUATION}

There exist for the moment transfer functions for three positions of the pump, fig. 4. It is of course possible to derive transfer functions for an infinite number of positions, at the expense of a considerable amount of work [8].

In earlier works, a few different faults have been studied: a cavitated valve plate, broken piston rings, damaged bearing etc [3]. This work has concentrated on the valve plate, and to make it possible to study the problem both computationally and experimentally, different designs of the pressure relief groove have been used (cf fig. 5), since this is geometrically measurable, and the leakage is reasonably comparable to damages caused by cavitation. The aim has been to introduce an internal leakage that is to be detected by vibration measurements earlier than it would be possible to detect it by efficiency measurements. The pressure relief groove has been altered to give $1,2, \ldots 9 \%$ loss in volumetric efficiency, and

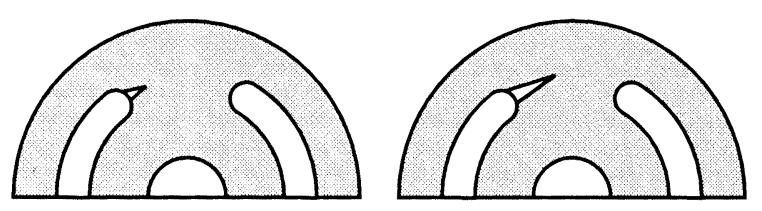

Figure 5. Schematic picture of one half of a valve plate with pressure relief groove. Left: normal groove. Right: groove giving efficiency loss. 


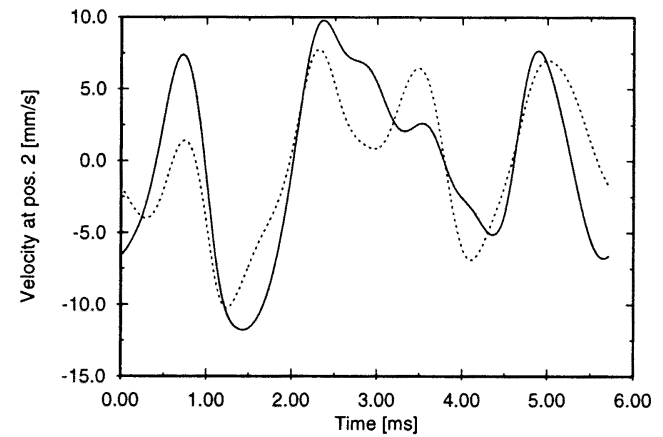

Figure 6. Vibration velocity at position 2 for original valve plate (solid line) and for valve plate giving $3 \%$ efficiency loss (dashed line).

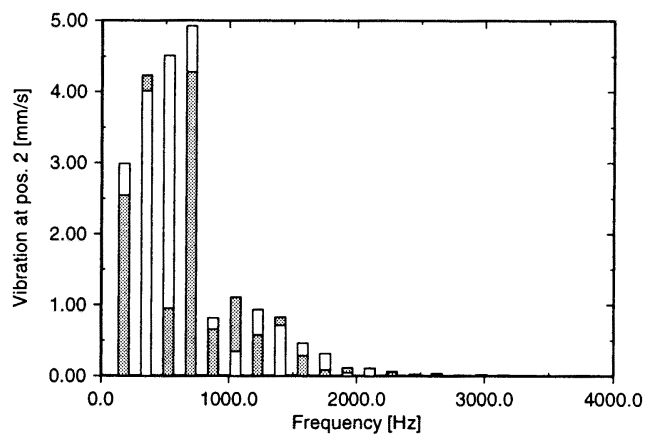

Figure 7. Amplitude of vibration velocity at position 2 for original valve plate (white) and for valve plate giving $3 \%$ efficiency loss (grey).

the surface vibration has been studied. For all the simulations the operating condition has been kept constant: rotational speed $=1500 \mathrm{rpm}$ and system pressure $=20 \mathrm{MPa}$.

In fig. 6 and 7 vibration velocity is shown for two cases for position 2 at the pump housing: the original valve plate and the valve plate giving a $3 \%$ efficiency loss. In fig. 6 the time signal is shown, whereas in fig. 7 the amplitude of the frequency spectrum of the signal is shown. It can be seen that there is an obvious difference in the signal between the two conditions, which is probably difficult to detect in the time signal but easier in the amplitude. However, if we go on to look at the case with $6 \%$ efficiency loss, fig. 8 , we can see that some of the changes have become smaller again, which may be more difficult to predict.

\section{Neural network training}

The evaluation of this problem has been made as follows: the original valve plate, and the plates giving $3 \%, 6 \%$ and $9 \%$ efficiency loss, respectively, have been used to train a neural network. The other cases have been used to evaluate the network: the $1 \%$ plate should be similar to the original plate, the $2 \%$ plate

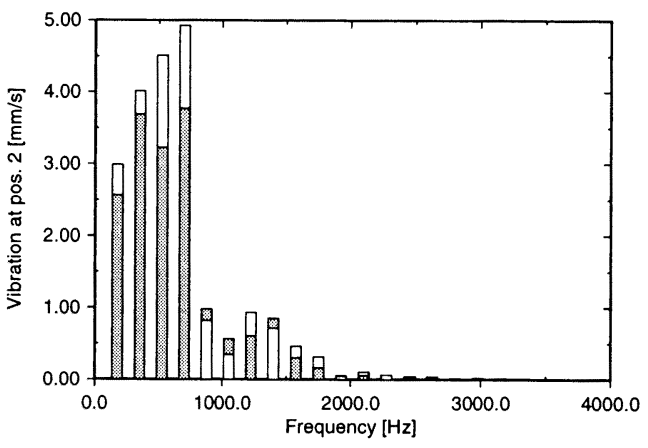

Figure 8. Amplitude of vibration velocity at position 2 for original valve plate (white) and for valve plate giving $6 \%$ efficiency loss (grey).

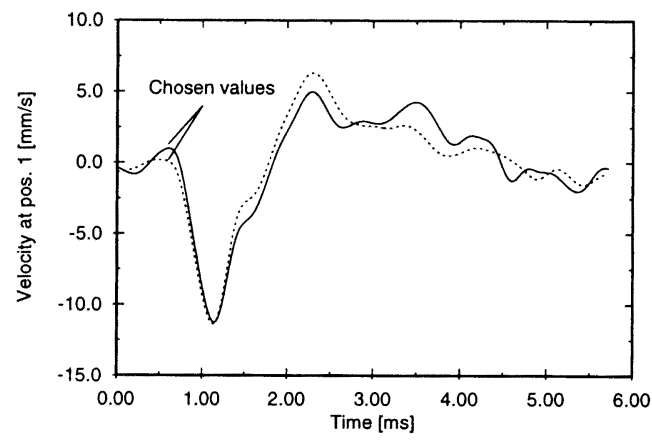

Figure 9 . Velocity at the pump surface at position 1 for original valve plate (solid line) and valve plate giving $5 \%$ efficiency loss (dashed line).

should be similar to the $3 \%$ valve plate, etc. In this case, the properties used as inputs to the neural net were chosen manually, by choosing properties that gave obvious differences between the four cases used for the training. These differences were monotonously increasing with increasing damages. The chosen properties were the peak of the velocity at position one as shown by the arrows in fig. 9, the fourth harmonic of the spectrum of the velocity amplitude at position 2 and 3 (cf fig. 10) and the fifth and sixth value of the velocity phase angle at position 2 (cf fig. 11). That makes five values from which to form an input vector. The values are normalised to give inputs to the neural net that are within the limits $-1 \lesssim i_{i} \lesssim 1$.

The network has, consequently, five input neurons. It also has a hidden layer, with 15 neurons (chosen because it turned out to work well), and an output layer with four neurons (one for each of the four conditions (valve plates) used for training). The transfer functions of the output layers are chosen according to eq. 2 , to ensure that the outputs stay within the limits $-1<i_{i}<1$ as desired according to fig. 3. After approximately 165 training epochs, the 


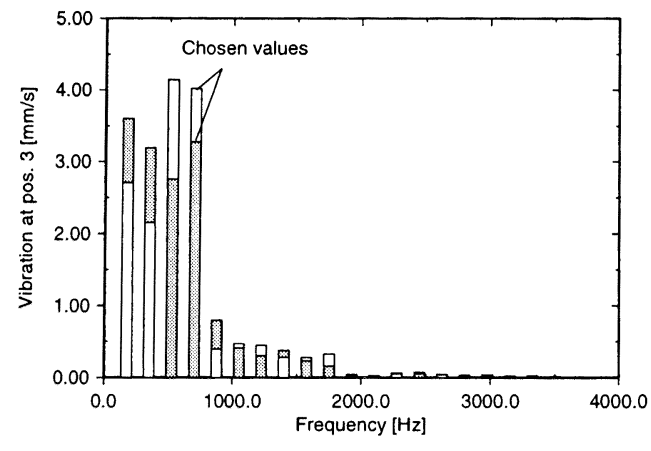

Figure 10. Velocity amplitude at the pump surface at position 3 for original valve plate (white bars) and valve plate giving $5 \%$ efficiency loss (grey bars).

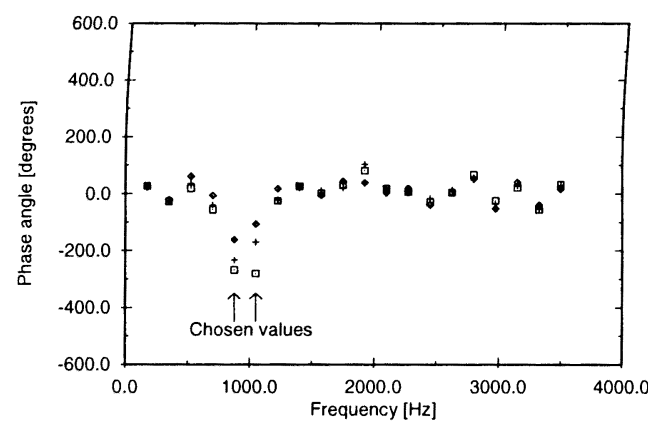

Figure 11. Velocity phase at the pump surface at position 2 . Shown is phase difference between original valve plate and the valve plates giving $3 \%(\diamond), 6 \%(+)$ and $9 \%(\bullet)$ efficiency loss, respectively.

network is trained acceptably. The acceptance level is set to a sum-squared error of less than 0.1. The net is trained with the weights randomly initialised. This means that two consecutive net trainings usually do not give exactly the same resulting net, since the solutions of the optimisation problem will differ.

\section{Neural network testing}

The next step is to test the nets with simulation results from the valve plates that have not been used for the training. The expected output values for those valve plates and the actual output values from five consecutive trainings are shown in table 1 . If a value is greater than 0.5 , it is considered to indicate that it should be close to 1 , and a value less than 0.5 is considered to indicate that it should be close to 0 . It can be seen that for most of the cases, the expected results are obtained. There are, however, three cases where the results are not as unequivocal as in the other cases: the last test condition, $8 \%$ efficiency loss, is three times recognised to be close both to the $9 \%$ and the $6 \%$ condition. This is, though, not too big a problem: the leakage is already large, and thus it is most important to detect that there is a large leakage, and that is certainly done. It is worth noting that for all cases the second largest value indicates the second closest condition ( $\mathrm{i}$ e for the $1 \%$ condition, the second value is considerably higher than the third and fourth, showing that condition two is not too far away).

\section{DISCUSSION}

In this paper we have used the time signal and the amplitude and the phase of the vibration signal of the pump housing for condition monitoring. There are of course other possibilities, such as measuring other parameters, e g flow, pressure, temperature etc. Other works (e g $[1,9])$ have shown, though, that the vibration signal contains much information. A combination of more parameters may be a good compromise, even though this means more parameters to take care of and thus a more complex problem to solve.

We have chosen to study time, amplitude and phase characteristics of the signal. This is to get as much information as possible out of it. It would certainly be valuable to also include wavelets in the process. In the time signal, it is possible to see that the pressurisation of the cylinder is made considerably faster for the cases with large leakage. Since this study is made on simulation results, measurement noise is not included, and, consequently, it is not too complicated to find the phase information. It is, though, not that simple with actual measurements, and this must of course be considered when implementing this kind of condition monitoring system.

We have been looking at three positions of the pump housing for the vibration measurements. This is because these transfer functions were already derived and verified. It is most likely that there are other positions that could give better results for the condition monitoring process, and also that there is one single position that could do the work; the transfer functions were derived for other purposes.

\section{CONCLUSIONS}

It has been shown that, by using the described condition monitoring technique, it is possible to detect the different leakage problems introduced in this paper. Neural networks are of good help in the process. It is indeed possible to estimate the degree of leakage for the non-trained cases. Due to the fact that there are verified transfer functions for the transition between internal forces and housing vibration it has been possible to use simulations instead of measurements. 
Table 1. Results from neural network training

\begin{tabular}{|c|c|c|c|c|c|c|c|c|c|c|}
\hline \multirow{2}{*}{$\begin{array}{l}\text { Output } \\
\text { number }\end{array}$} & \multicolumn{10}{|c|}{ Efficiency loss } \\
\hline & $0 \%$ & $1 \%$ & $2 \%$ & $3 \%$ & $4 \%$ & $5 \%$ & $6 \%$ & $7 \%$ & $8 \%$ & $9 \%$ \\
\hline \multicolumn{11}{|c|}{ The output vectors that the nets are trained for } \\
\hline 1 & 1 & & & 0 & & & 0 & & & 0 \\
\hline 2 & 0 & & & 1 & & & 0 & & & 0 \\
\hline 3 & 0 & & & 0 & & & 1 & & & 0 \\
\hline 4 & 0 & & & 0 & & & 0 & & & 1 \\
\hline \multicolumn{11}{|c|}{ Expected approximate values for the test cases } \\
\hline 1 & & 1 & 0 & & 0 & 0 & & 0 & 0 & \\
\hline 2 & & 0 & 1 & & 1 & 0 & & 0 & 0 & \\
\hline 3 & & 0 & 0 & & 0 & 1 & & 1 & 0 & \\
\hline 4 & & 0 & 0 & & 0 & 0 & & 0 & 1 & \\
\hline \multicolumn{11}{|c|}{ Test results from test case 1} \\
\hline 1 & & 0.9162 & 0.2799 & & 0.0063 & 0.0013 & & 0.0008 & 0.0008 & \\
\hline 2 & & 0.1713 & 0.7881 & & 0.7834 & 0.1659 & & 0.0271 & 0.0272 & \\
\hline 3 & & 0.0772 & 0.0569 & & 0.4414 & 0.8460 & & 0.6502 & 0.3935 & \\
\hline 4 & & 0.0007 & 0.0004 & & 0.0005 & 0.0059 & & 0.3281 & 0.6960 & \\
\hline \multicolumn{11}{|c|}{ Test results from test case 2} \\
\hline 1 & & 0.9109 & 0.3000 & & 0.0058 & 0.0024 & & 0.0022 & 0.0022 & \\
\hline 2 & & 0.1892 & 0.8830 & & 0.7239 & 0.2513 & & 0.0221 & 0.0132 & \\
\hline 3 & & 0.0534 & 0.0191 & & 0.3803 & 0.7958 & & 0.7841 & 0.6019 & \\
\hline 4 & & 0.0009 & 0.0007 & & 0.0045 & 0.0171 & & 0.3329 & 0.6870 & \\
\hline \multicolumn{11}{|c|}{ Test results from test case 3} \\
\hline 1 & & 0.9552 & 0.1444 & & 0.0015 & 0.0007 & & 0.0007 & 0.0006 & \\
\hline 2 & & 0.0499 & 0.7606 & & 0.7951 & 0.2788 & & 0.0244 & 0.0097 & \\
\hline 3 & & 0.0308 & 0.0495 & & 0.3824 & 0.8291 & & 0.6331 & 0.3755 & \\
\hline 4 & & 0.0040 & 0.0031 & & 0.0035 & 0.0139 & & 0.2935 & 0.6812 & \\
\hline \multicolumn{11}{|c|}{ Test results from test case 4} \\
\hline 1 & & 0.8642 & 0.2576 & & 0.0184 & 0.0061 & & 0.0033 & 0.0031 & \\
\hline 2 & & 0.1227 & 0.8529 & & 0.6995 & 0.1994 & & 0.0429 & 0.0330 & \\
\hline 3 & & 0.0398 & 0.0464 & & 0.3849 & 0.8211 & & 0.7577 & 0.5592 & \\
\hline 4 & & 0.0039 & 0.0020 & & 0.0050 & 0.0206 & & 0.2942 & 0.6384 & \\
\hline \multicolumn{11}{|c|}{ Test results from test case 5} \\
\hline 1 & & 0.9454 & 0.3092 & & 0.0032 & 0.0003 & & 0.0002 & 0.0002 & \\
\hline 2 & & 0.1106 & 0.7821 & & 0.7667 & 0.1940 & & 0.0459 & 0.0384 & \\
\hline 3 & & 0.0515 & 0.0436 & & 0.3719 & 0.8127 & & 0.7644 & 0.5674 & \\
\hline 4 & & 0.0043 & 0.0005 & & 0.0013 & 0.0147 & & 0.2636 & 0.5673 & \\
\hline
\end{tabular}

\section{REFERENCES}

[1] J Esser. Diagnosesystem für Hydraulikpumpen. $O+P$, Ölhydraulik und Pneumatik, 36(3):176-181, 1992.

[2] T Ramdén, K G Weddfelt and J-O Palmberg. Condition monitoring of fluid power pumps by vibration measurement. In $\mathrm{N}$ Way, editor, 10th International Conference on Fluid Power - the future for hydraulics, pages 263-276, London, 1993. BHR Group Ltd, Mech Eng Publications Ltd.

[3] T Ramdén, $\mathrm{P}$ Krus and J-O Palmberg. Condition monitoring of fluid power pumps using vibration measurements and neural networks trained with complex optimisation. In Vibration and Noise'95, Stafford, UK, 1995. Staffordshire Univ.

[4] J Hertz, A Krogh and R G Palmer. Introduction to the Theory of Neural Computation. Addison-Wesley Publishing Company, Redwood City, CA, 1991.

[5] H Demuth and M Beale. Neural Network Toolbox User's Guide. The MathWorks, Inc., Massachusetts, 1993.
[6] M Pettersson. Design of Fluid Power Piston Pumps - With Special Reference to Noise Reduction. PhD thesis, Linköping Univ, Dept of Mech Eng, S-581 83 Linköping, Sweden, 1995.

[7] Hopsan. Hopsan - a Simulation Package, User's Guide. Dept of Mech Eng, Linköping Univ, S-581 83 Linköping, Sweden, 1991.

[8] M Pettersson, K Weddfelt and J-O Palmberg. Prediction of structural and audible noise from axial piston pumps using transfer functions. In Eighth Bath International Fluid Power Workshop on Design and Performance, Bath, UK, 1995.

[9] R J Kuoppala, E O Jantunen and P A Enwald. Condition monitoring methods for rotating machinery. In K. Holmberg and A. Folkeson, editors, Operational Reliability and Systematic Maintenance, chapter 3.2, pages 175-198. Elsevier Science Publishers Ltd, Essex, UK, 1991. 